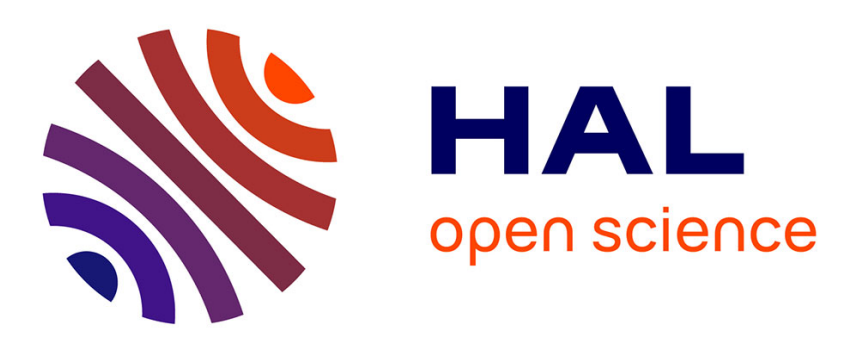

\title{
Sum-capacity of Uplink Multiband Satellite Communications with Nonlinear Impairments
}

\author{
Arthur Louchart, Philippe Ciblat, Charly Poulliat
}

\section{To cite this version:}

Arthur Louchart, Philippe Ciblat, Charly Poulliat. Sum-capacity of Uplink Multiband Satellite Communications with Nonlinear Impairments. IEEE International Conference on Communications (ICC), 2021, Montréal, Canada. hal-03291059

\section{HAL Id: hal-03291059 \\ https://hal.science/hal-03291059}

Submitted on 19 Jul 2021

HAL is a multi-disciplinary open access archive for the deposit and dissemination of scientific research documents, whether they are published or not. The documents may come from teaching and research institutions in France or abroad, or from public or private research centers.
L'archive ouverte pluridisciplinaire HAL, est destinée au dépôt et à la diffusion de documents scientifiques de niveau recherche, publiés ou non, émanant des établissements d'enseignement et de recherche français ou étrangers, des laboratoires publics ou privés. 


\section{Sum-capacity of Uplink Multiband Satellite Communications with Nonlinear Impairments}

\author{
Arthur Louchart \\ Telecom Paris \\ Institut Polytechnique de Paris \\ Palaiseau, France \\ arthur.louchart@telecom-paris.fr
}

\author{
Philippe Ciblat \\ Telecom Paris \\ Institut Polytechnique de Paris \\ Palaiseau, France \\ philippe.ciblat@telecom-paris.fr
}

\author{
Charly Poulliat \\ INP-ENSEEIHT \\ Toulouse, France \\ charly.poulliat@enseeiht.fr
}

\begin{abstract}
A compact and closed-form expression of capacity is derived for a uplink multiband satellite system in the presence of nonlinear interference. The nonlinear effect comes from the satellite high-power amplifier modeled by a Volterra series expansion. The derivations reveal that the nonlinear interference can provide a constructive power contribution that could be used to increase the transmission rate. Consequently, decoders designed by viewing this interference as only an additional noise are suboptimal. Numerical results confirm this claim and also shows that an appropriate power allocation amongst the subbands may be of interest.
\end{abstract}

Index Terms - High power amplifier, Volterra series, nonlinear impairment, capacity, multiband, uplink satellite communication.

\section{INTRODUCTION}

Satellite communications in addition to next generation cellular systems $(5 \mathrm{G} / 6 \mathrm{G})$ will become a key component to sustain the exponential growth of the Internet traffic. As in [1], it is envisioned to open Ka-band to satellite communications for new services for both forward and return links. In this paper, we focus on the uplink/return link where non-colocated terrestrial transmitters send their data stream to the same satellite, which forwards the aggregate stream into a terrestrial gateway seen as the final receiver. For the uplink to the satellite, the users belong to the same beam but are separated into different subbands and different timeslots (by using the so-called Multiple Frequency - Time Division Multiple Access scheme, MF-TDMA) in order to avoid interference.

Actually, the above-mentioned interference does not exist if the satellite transponder always operates into the linear regime of the satellite amplifier. Here we assume that the transponder is single and works on the aggregate signal created by all the subbands. When operating in the nonlinear regime of the amplifier, in-band and out-of-band distortions are generated causing a loss in performance. In order to limit these drawbacks, we can proceed in three different ways:

- We force the system to work into the linear regime by applying an Input Back-Off (IBO). However, the energy efficiency of the amplifier becomes lower. More importantly, the maximum sum-capacity of the uplink system

This work has been funded by ANR through the SIERRA FrenchLuxembourgian grant. is not reached by fixing an operating point preventing nonlinearity to disturb the incoming signal.

- When operating in the non linear regime, precompensation methods (see [2] and reference therein) can be applied in order to mitigate nonlinear interference at the receiver side. However, in uplink, this approach can be only implemented in a distributed manner and, thus, it is not optimal anymore. Indeed, one are not able to cancel all the nonlinear impairments since only in-band impairments can be pre-compensated for.

- Assuming the nonlinear regime, post-compensation at the end receiver (i.e. at the gateway in our uplink scenario) can be carried out.

In this paper, we aim to evaluate theoretically the system sum-capacity when operating in the nonlinear regime, in order to evaluate clearly the system capacity loss with respect to the case where the nonlinear interference is seen as an additional noise, as well as in order to investigate on the impact of the power allocation amongst the subbands. To do so, we derive a closed-form expression for the sum-capacity of the uplink satellite communication system when nonlinearity occurs. We do not assume pre-compensation as well as post-compensation. The main contributions can be summarized as follows:

- First, the impact of the satellite amplifier is modeled through the use of Volterra series expansion of the received signal. While this model has been deeply investigated in [3], we propose here an alternative and elegant approach to derive analytically all required terms. Indeed, the proposed derivations for the sum-capacity will rely on the closed-form derivations of the auto-correlation of the additional nonlinear term as well as the crosscorrelation of this nonlinear term and the linear one. In the context of multiband communications, these terms have been characterized by [3]. Nevertheless, the existing characterization is tedious and does not provide clear insights. Here we propose to follow an alternative path to obtain much more compact and insightful closed-form expressions for these terms. We especially prove that they are all posynomials with respect to the subband powers. This property may be useful for future resource allocation management [4]. 
- Based on these new expressions, we are able to derive closed-form expressions for the sum-capacity for two decoding strategies. First, we consider the optimal case for which the decoder takes into account the signal nonlinear structure. Then, we consider the case of a nonlinearity-agnostic decoder, for which any term coming from the nonlinearity is considered as an additional noise.

The rest of the paper is organized as follows: the system model of the uplink multiband satellite communications system with nonlinearity due to the satellite amplifier is given in Section II. The general expressions for the sum-capacity associated with both decoding strategies are derived in Section III. Then the correlation terms involved in the sum-capacity are expressed in a compact closed-form in Section IV. Numerical results are presented in Section V. Finally, concluding remarks and perspectives are drawn in Section VI.

\section{SySTEM MODEL}

We consider a uplink multiband satellite communication system composed of an high Power Amplifier (HPA), Input Multiplexer (IMUX) and Output Multiplexer (OMUX) [2], [3]. The two last devices are assumed to be ideal.

We consider $K$ terrestrial users using adjacent bands in a single beam (FDMA). User $k, k=1 \cdots K$, transmits an independent and identically-distributed symbol sequence $\left\{a_{k, n}\right\}_{n \in \mathbb{Z}}$ towards the satellite. All users have the same shaping filter with an impulse response $p_{T}(t)$. For the sake of simplicity, $p_{T}(t)$ is assumed to be a square-root raised cosine (RRC) with roll-off $r$.

We denote by $u_{k}(t)$ the baseband signal sent by user $k$,

$$
u_{k}(t)=\sum_{n \in \mathbb{Z}} a_{k, n} p_{T}\left(t-n T_{s}\right)
$$

where $T_{s}$ is the symbol period. Each signal $x_{k}(t)$ is shifted to frequency $f_{k}$. In order to avoid subband interference, the difference between two adjacent frequencies, denoted by $\Delta F$, is equal to $\Delta F=(1+r) / T_{s}$.

The satellite receives the carrier signal $x_{c}(t)$ equal to the sum of the $K$-transposed signals, as follows

$$
x_{c}(t)=\sum_{k=1}^{K} \sqrt{G_{k}} u_{k}(t) e^{2 i \pi f_{k} t} .
$$

with $G_{k}$ the channel gain between user $k$ and the satellite.

Let $y_{c}(t)$ be the received signal at the gateway. This signal has undergone the disturbance from the satellite amplifier, modeled by the following third-order Volterra series expansion, as well as noise added at the receiver. The link between the satellite and the gateway is assumed ideal [3],

$$
y_{c}(t)=\gamma_{1} x_{c}(t)+\gamma_{3} x_{c}(t) x_{c}(t) x_{c}^{*}(t)+w_{c}(t),
$$

where $\gamma_{1}$ and $\gamma_{3}$ are fixed parameters depending on the HPA and $w_{c}(t)$ is an additive white zero-mean Gaussian noise. The superscript $(.)^{*}$ stands for complex conjugate.

At the receiver side, to recover the bits stream of user $k$, we need to demodulate $y_{c}(t)$ around frequency $f_{k}$, as follows

$$
y_{k}(t)=y_{c}(t) e^{-2 i \pi f_{k} t} \text {. }
$$

After matched filtering using $p_{R}(t):=p_{T}^{*}(-t)$, we have

$$
z_{k}(t)=\int_{\mathbb{R}} p_{R}(\tau) y_{k}(t-\tau) d \tau
$$

Finally the signal $z_{k}(t)$ is sampled at the symbol rate, leading to the following sequence of samples $z_{k, n}=z_{k}\left(n T_{s}\right)$ that are used for detection and decoding.

The signal $z_{k, n}$ can be decomposed into three terms

$$
z_{k, n}=z_{k, n}^{\mathrm{L}}+z_{k, n}^{\mathrm{NL}}+w_{k, n}
$$

where

- $z_{k, n}^{\mathrm{L}}$ depends linearly on the symbols $\left\{a_{k, n}\right\}_{k, n}$,

- $z_{k, n}^{\mathrm{NL}}$ depends nonlinearly on the symbols $\left\{a_{k, n}\right\}_{k, n}$,

- $w_{k, n}:=\int_{\mathbb{R}} p_{R}(\tau) w_{c}\left(n T_{s}-\tau\right) e^{-2 i \pi f_{k}\left(n T_{s}-\tau\right)} d \tau$ is a white zero-mean Gaussian noise with variance $\mathcal{P}_{\mathrm{W}}$.

To obtain closed-form expressions for both terms $z_{k, n}^{\mathrm{L}}$ and $z_{k, n}^{\mathrm{NL}}$ in Eq. (6), Eqs. (1)-(2) are first inserted in Eq. (3). Then, after frequency down-shifting and matched filtering, the signal term $z_{k}(t)$ writes as follows

$$
\begin{aligned}
z_{k}(t) & =\gamma_{1} \sum_{k^{\prime}=1}^{K} \sum_{n^{\prime} \in \mathbb{Z}} a_{k^{\prime}, n^{\prime}} \sqrt{G_{k^{\prime}}} e^{2 i \pi\left(k^{\prime}-k\right) \Delta F t} \\
& \times h_{1}\left(t-n^{\prime} T_{s}, k^{\prime}-k\right) \\
& +\gamma_{3} \sum_{k_{1}, k_{2}, k_{3}=1}^{K} \sum_{n_{1}, n_{2}, n_{3} \in \mathbb{Z}} a_{k_{1}, n_{1}} a_{k_{2}, n_{2}} a_{k_{3}, n_{3}}^{*} \\
& \times \sqrt{G_{k_{1}} G_{k_{2}} G_{k_{3}}} e^{2 i \pi\left(k_{1}+k_{2}-k_{3}-k\right) \Delta F t} \\
& \times h_{3}\left(t-n_{1} T_{s}, t-n_{2} T_{s}, t-n_{3} T_{s}, k_{1}+k_{2}-k_{3}-k\right) \\
& +\int_{\mathbb{R}} p_{R}(\tau) w_{c}(t-\tau) e^{-2 i \pi f_{k}(t-\tau)} d \tau,
\end{aligned}
$$

with

$$
h_{1}\left(t_{1}, \ell\right)=\int_{\mathbb{R}} p_{T}\left(t_{1}-\tau\right) p_{R}(\tau) e^{-2 i \pi \ell \Delta F \tau} d \tau
$$

and

$$
\begin{aligned}
h_{3}\left(t_{1}, t_{2}, t_{3}, \ell\right) & =\int_{\mathbb{R}} p_{T}\left(t_{1}-\tau\right) p_{T}\left(t_{2}-\tau\right) \\
& \times p_{T}\left(t_{3}-\tau\right) p_{R}(\tau) e^{-2 i \pi \ell \Delta F \tau} d \tau .
\end{aligned}
$$

By sampling $z_{k}(t)$ at time $n T_{s}$, the linear part is

$$
\begin{aligned}
z_{k, n}^{\mathrm{L}} & =\gamma_{1} \sum_{k^{\prime}=1}^{K} \sum_{n^{\prime} \in \mathbb{Z}} a_{k^{\prime}, n-n^{\prime}} \sqrt{G_{k^{\prime}}} e^{2 i \pi\left(k^{\prime}-k\right) \Delta F n T_{s}} \\
& \times h_{1}\left(n^{\prime} T_{s}, k^{\prime}-k\right),
\end{aligned}
$$

and the nonlinear part is

$$
\begin{aligned}
z_{k, n}^{\mathrm{NL}} & =\gamma_{3} \sum_{k_{1}, k_{2}, k_{3}=1}^{K} \sum_{n_{1}, n_{2}, n_{3} \in \mathbb{Z}} a_{k_{1}, n-n_{1}} a_{k_{2}, n-n_{2}} a_{k_{3}, n-n_{3}}^{*} \\
& \times \sqrt{G_{k_{1}} G_{k_{2}} G_{k_{3}}} e^{2 i \pi\left(k_{1}+k_{2}-k_{3}-k\right) \Delta F n T_{s}} \\
& \times h_{3}\left(n_{1} T_{s}, n_{2} T_{s}, n_{3} T_{s}, k_{1}+k_{2}-k_{3}-k\right) .
\end{aligned}
$$

Notice that as $p_{T}(t)$ is a square-root Nyquist filter with respect to $T_{s}$, the sequence $n \mapsto h_{1}\left(n T_{s}, k\right)$ is zero for any 
$n \neq 0$. In addition as $\Delta F$ is equal to the bandwidth of $p_{T}(t)$, the sequence $k \mapsto h_{1}\left(n T_{s}, k\right)$ is zero for any $k \neq 0$. Consequently, in Eq. (9), we have $n^{\prime}=n$ and $k^{\prime}=k$ which implies that

$$
z_{k, n}^{\mathrm{L}}=\gamma_{1} \sqrt{G_{k}} a_{k, n},
$$

since $h_{1}(0,0)=1$ by the filter energy normalization.

\section{CAPACITY CLOSED-FORM EXPRESSIONS IN NONLINEAR REGIME}

In this section, we provide a closed-form expression for the mutual information when assuming the input signal as a Gaussian random process and when we neglect the time and frequency correlations between the linear part and the nonlinear part (i.e., we consider that $z_{k, n}^{\mathrm{L}}$ and $z_{k^{\prime}, n^{\prime}}^{\mathrm{NL}}$ with $k \neq k^{\prime}$ or $n \neq n^{\prime}$ are uncorrelated). Using a slight abuse of language, this mutual information will be called capacity in the remainder of the paper.

The input-output link (modeling the uplink channel, the satellite amplifier, and the noise at the receiver end) is given by Eq. (6) where $z_{k, n}^{\mathrm{L}}$ and $z_{k, n}^{\mathrm{NL}}$ are given by Eq. (11) and Eq. (10) respectively.

Assuming a complex-valued circularly-symmetric Gaussian random variable $a$ as the channel input and $z$ the output channel, the capacity is given by [5]

$$
C=\log _{2}\left(1+\frac{\mathbb{E}\left[a z^{*}\right] \mathbb{E}\left[z a^{*}\right]}{\mathbb{E}\left[|a|^{2}\right] \mathbb{E}\left[z z^{*}\right]-\mathbb{E}\left[a z^{*}\right] \mathbb{E}\left[z a^{*}\right]}\right) .
$$

For applying Eq. (12) to our system, we assume that

- $\left\{a_{k, n}\right\}_{k, n}$ are iid complex-valued circularly-symmetric Gaussian random process,

- and as already said, that $z_{k, n}$ is an uncorrelated sequence.

Reminding Eqs (6),(11) and Eq. (12), the capacity for user $k$, denoted by $C(k)$, becomes

$$
C(k)=\log _{2}(1+Q(k))
$$

with

$$
Q(k)=\frac{\mathcal{P}_{\mathrm{L}}^{2}(k)+2 \mathcal{P}_{\mathrm{L}}(k) \Re\left\{\mathcal{P}_{\mathrm{LNL}}(k)\right\}+\left|\mathcal{P}_{\mathrm{LNL}}(k)\right|^{2}}{\mathcal{P}_{\mathrm{L}}(k) \mathcal{P}_{\mathrm{NL}}(k)+\mathcal{P}_{\mathrm{L}}(k) \mathcal{P}_{\mathrm{W}}-\left|\mathcal{P}_{\mathrm{LNL}}(k)\right|^{2}},
$$

where

- $\mathcal{P}_{\mathrm{L}}(k)=\mathbb{E}\left[\left|z_{k, n}^{\mathrm{L}}\right|^{2}\right]$ is the auto-correlation of the linear part,

- $\mathcal{P}_{\mathrm{NL}}(k)=\mathbb{E}\left[\left|z_{k, n}^{\mathrm{NL}}\right|^{2}\right]$ is the auto-correlation of the nonlinear part, and

- $\mathcal{P}_{\mathrm{LNL}}(k)=\mathbb{E}\left[z_{k, n}^{\mathrm{L}} z_{k, n}^{\mathrm{NL}}\right]$ is the cross-correlation between the linear and nonlinear parts.

As a first remark, one can note that although there is a negative sign in the denominator of $Q(k)$, the term is positive. Indeed, according to Cauchy-Schwartz inequality, we have $\left|\mathbb{E}\left[z_{k, n}^{\mathrm{L}} z_{k, n}^{\mathrm{NL}}\right]\right|^{2} \leq \mathbb{E}\left[\left|z_{k, n}^{\mathrm{L}}\right|^{2}\right] \mathbb{E}\left[\left|z_{k, n}^{\mathrm{NL}}\right|^{2}\right]$ which is equivalent to $\left|\mathcal{P}_{\mathrm{LNL}}(k)\right|^{2} \leq \mathcal{P}_{\mathrm{L}}(k) \mathcal{P}_{\mathrm{NL}}(k)$. As a second remark, the cross-correlation plays a significant role in the capacity. If $\Re\left\{\mathcal{P}_{\text {LNL }}(k)\right\}>0$, the capacity is higher than the case $\Re\left\{\mathcal{P}_{\mathrm{LNL}}(k)\right\}=0$. In addition, the capacity does not vanish even if $\mathcal{P}_{\mathrm{L}}(k)=0$, since the nonlinear term provides information on the current symbol. Notice that in [3], only the following Mean Square Error (MSE) is analyzed ${ }^{1}$

$$
\mathbb{E}\left[\left|z_{k, n}-\gamma_{1} \sqrt{G_{k}} a_{k, n}\right|^{2}\right]=\mathcal{P}_{\mathrm{NL}}(k)+\mathcal{P}_{\mathrm{W}} .
$$

which does not capture the role of the cross-correlation.

We now assume that the decoder sees the nonlinear interference as noise, as currently done in most receivers, and so is not adapted to the presence of nonlinearity. As a consequence, the corresponding capacity is obtained as follows

$$
\underline{C}(k)=\log _{2}(1+\underline{Q}(k))
$$

with

$$
\underline{Q}(k)=\frac{\mathcal{P}_{\mathrm{L}}(k)}{\mathcal{P}_{\mathrm{NL}}(k)+\mathcal{P}_{\mathrm{W}}},
$$

where we just have to calculate the Signal-to-Interferenceplus-Noise Ratio (SINR). One can remark that Eq. (14) is equal to Eq. (13) by forcing $\mathcal{P}_{\mathrm{LNL}}(k)=0$. Thus if the cross-correlation vanishes, the nonlinear interference purely corresponds to a degradation. If not, taking into account the cross-correlation at the decoder side is of interest.

\section{AUTO-CORRELATION AND CROSS-CORRELATION CLOSED FORM EXPRESSIONS IN NONLINEAR REGIME}

The goal of this Section is to provide compact and insightful closed-form expressions for $\mathcal{P}_{\mathrm{L}}(k), \mathcal{P}_{\mathrm{NL}}(k)$, and $\mathcal{P}_{\mathrm{LNL}}(k)$.

\section{A. Derivations of $\mathcal{P}_{\mathrm{L}}(k)$}

According to Eq. (11), one can easily check that

$$
\mathcal{P}_{\mathrm{L}}(k)=\gamma_{1}^{2} G_{k} P_{k},
$$

with $P_{k}=\mathbb{E}\left[\left|a_{k, n}\right|^{2}\right]$ the transmit power of user $k$.

\section{B. Derivations of $\mathcal{P}_{\mathrm{NL}}(k)$}

According to Eq. (10), we obtain

$$
\begin{aligned}
& \mathcal{P}_{\mathrm{NL}}(k)=\gamma_{3}^{2} \sum_{k_{1}, k_{2}, k_{3}=1}^{K} \sum_{k_{1}^{\prime}, k_{2}^{\prime}, k_{3}^{\prime}=1}^{K} \sum_{n_{1}, n_{2}, n_{3} \in \mathbb{Z}} \sum_{n_{1}^{\prime}, n_{2}^{\prime}, n_{3}^{\prime} \in \mathbb{Z}} \\
& \sqrt{G_{k_{1}} G_{k_{2}} G_{k_{3}} G_{k_{1}^{\prime}} G_{k_{2}^{\prime}} G_{k_{3}^{\prime}}} \\
& \times e^{2 i \pi\left(k_{1}+k_{2}-k_{3}-k\right) \Delta F n T_{s}} e^{-2 i \pi\left(k_{1}^{\prime}+k_{2}^{\prime}-k_{3}^{\prime}-k\right) \Delta F n T_{s}} \\
& \times \mathbb{E}\left[a_{k_{1}, n-n_{1}} a_{k_{2}, n-n_{2}} a_{k_{3}, n-n_{3}}^{*} a_{k_{1}^{\prime}, n-n_{1}^{\prime}}^{*} a_{k_{2}^{\prime}, n-n_{2}^{\prime}} a_{k_{3}^{\prime}, n-n_{3}^{\prime}}\right] \\
& \times h_{3}\left(n_{1} T_{s}, n_{2} T_{s}, n_{3} T_{s}, k_{1}+k_{2}-k_{3}-k\right) \\
& \times h_{3}^{*}\left(n_{1}^{\prime} T_{s}, n_{2}^{\prime} T_{s}, n_{3}^{\prime} T_{s}, k_{1}^{\prime}+k_{2}^{\prime}-k_{3}^{\prime}-k\right) .
\end{aligned}
$$

This term is already involved in the derivations done in [3]. Nevertheless, the expressions provided in [3] is quite complicated and spread over the paper (see Tables 1 and 2, Eqs. (17) and (20) in [3]). Here, we propose a new way to derive $\mathcal{P}_{\mathrm{NL}}(k)$ in order to obtain a more compact and insightful form. So, unlike [3], we start by managing the

\footnotetext{
${ }^{1}$ Actually, the cross correlation in [3] occurred since the author considered that $h_{1}\left(n T_{s}, k\right)$ may not vanish for non-zero $n$ or $k$. But under our orthogonality assumption, the cross-correlation term disappears in the MSE.
} 
term $\mathbb{E}\left[a_{k_{1}, n-n_{1}} a_{k_{2}, n-n_{2}} a_{k_{3}, n-n_{3}}^{*} a_{k_{1}^{\prime}, n-n_{1}^{\prime}}^{*} a_{k_{2}^{\prime}, n-n_{2}^{\prime}}^{*} a_{k_{3}^{\prime}, n-n_{3}^{\prime}}\right]$ and then the term $h_{3}$.

As $\left\{a_{k, n}\right\}_{k, n}$ are circularly-symmetric complex-valued Gaussian random process, we have (see (1.68) in [6])

$$
\begin{aligned}
& \mathbb{E}\left[a_{k_{1}, n-n_{1}} a_{k_{2}, n-n_{2}} a_{k_{3}, n-n_{3}}^{*} a_{k_{1}^{\prime}, n-n_{1}^{\prime}}^{*} a_{k_{2}^{\prime}, n-n_{2}^{\prime}}^{*} a_{k_{3}^{\prime}, n-n_{3}^{\prime}}\right]= \\
& \mathbb{E}\left[a_{k_{1}, n-n_{1}} a_{k_{1}^{\prime}, n-n_{1}^{\prime}}^{*}\right] \mathbb{E}\left[a_{k_{2}, n-n_{2}} a_{k_{3}, n-n_{3}}^{*}\right] \\
& \times \mathbb{E}\left[a_{k_{2}^{\prime}, n-n_{2}^{\prime}}^{*} a_{k_{3}^{\prime}, n-n_{3}^{\prime}}\right] \\
& +\mathbb{E}\left[a_{k_{1}, n-n_{1}} a_{k_{1}^{\prime}, n-n_{1}^{\prime}}^{*}\right] \mathbb{E}\left[a_{k_{2}, n-n_{2}} a_{k_{2}^{\prime}, n-n_{2}^{\prime}}^{*}\right] \\
& \times \mathbb{E}\left[a_{k_{3}, n-n_{3}}^{*} a_{k_{3}^{\prime}, n-n_{3}^{\prime}}\right] \\
& +\mathbb{E}\left[a_{k_{1}, n-n_{1}} a_{k_{3}, n-n_{3}}^{*}\right] \mathbb{E}\left[a_{k_{2}, n-n_{2}} a_{k_{2}^{\prime}, n-n_{2}^{\prime}}^{*}\right] \\
& \times \mathbb{E}\left[a_{k_{1}^{\prime}, n-n_{1}^{\prime}}^{*} a_{k_{3}^{\prime}, n-n_{3}^{\prime}}\right] \\
& +\mathbb{E}\left[a_{k_{1}, n-n_{1}} a_{k_{3}, n-n_{3}}^{*}\right] \mathbb{E}\left[a_{k_{2}, n-n_{2}} a_{k_{1}^{\prime}, n-n_{1}^{\prime}}^{*}\right] \\
& \times \mathbb{E}\left[a_{k_{2}^{\prime}, n-n_{2}^{\prime}}^{*} a_{k_{3}^{\prime}, n-n_{3}^{\prime}}\right] \\
& +\mathbb{E}\left[a_{k_{1}, n-n_{1}} a_{k_{2}^{\prime}, n-n_{2}^{\prime}}^{*}\right] \mathbb{E}\left[a_{k_{2}, n-n_{2}} a_{k_{3}, n-n_{3}}^{*}\right] \\
& \times \mathbb{E}\left[a_{k_{1}^{\prime}, n-n_{1}^{\prime}}^{*} a_{k_{3}^{\prime}, n-n_{3}^{\prime}}\right] \\
& +\mathbb{E}\left[a_{k_{1}, n-n_{1}} a_{k_{2}^{\prime}, n-n_{2}^{\prime}}^{*}\right] \mathbb{E}\left[a_{k_{2}, n-n_{2}} a_{k_{1}^{\prime}, n-n_{1}^{\prime}}^{*}\right] \\
& \times \mathbb{E}\left[a_{k_{3}, n-n_{3}}^{*} a_{k_{3}^{\prime}, n-n_{3}^{\prime}}\right]
\end{aligned}
$$

Notice that in [3] APSK constellation (instead of Gaussian one) is considered. Then a seventh term related to the sixorder cumulant of $\left\{a_{k, n}\right\}_{k, n}$ is required. Here the assumption on Gaussian constellation is done to resort to Eqs. (13)-(14).

As we have six additive terms in Eq. (17), Eq. (16) can be split into the six following terms

$$
\begin{aligned}
\mathcal{P}_{\mathrm{NL}}(k) & =p_{\mathrm{NL}}^{(1)}(k)+p_{\mathrm{NL}}^{(2)}(k)+p_{\mathrm{NL}}^{(3)}(k) \\
& +p_{\mathrm{NL}}^{(4)}(k)+p_{\mathrm{NL}}^{(5)}(k)+p_{\mathrm{NL}}^{(6)}(k) .
\end{aligned}
$$

It remains now to derive in closed-form each term $p_{\mathrm{NL}}^{(j)}(k)$ for $j=1, \cdots, 6$.

a) Derivations of $p_{\mathrm{NL}}^{(1)}(k)$ : According to Eqs. (17)-(16), we have

$$
\begin{aligned}
p_{\mathrm{NL}}^{(1)}(k)= & \gamma_{3}^{2} \sum_{k_{1}, k_{2}, k_{3}=1}^{K} \sum_{k_{1}^{\prime}, k_{2}^{\prime}, k_{3}^{\prime}=1}^{K} \sum_{n_{1}, n_{2}, n_{3} \in \mathbb{Z}} \sum_{n_{1}^{\prime}, n_{2}^{\prime}, n_{3}^{\prime} \in \mathbb{Z}} \\
& \sqrt{G_{k_{1}} G_{k_{2}} G_{k_{3}} G_{k_{1}^{\prime}} G_{k_{2}^{\prime}} G_{k_{3}^{\prime}}} \\
\times & e^{2 i \pi\left(k_{1}+k_{2}-k_{3}\right) \Delta F n T_{s}} e^{-2 i \pi\left(k_{1}^{\prime}+k_{2}^{\prime}-k_{3}^{\prime}\right) \Delta F n T_{s}} \\
\times & \mathbb{E}\left[a_{k_{1}, n-n_{1}} a_{k_{1}^{\prime}, n-n_{1}^{\prime}}^{*}\right] \mathbb{E}\left[a_{k_{2}, n-n_{2}} a_{k_{3}, n-n_{3}}^{*}\right] \\
\times & \mathbb{E}\left[a_{k_{2}^{\prime}, n-n_{2}^{\prime}}^{*} a_{k_{3}^{\prime}, n-n_{3}^{\prime}}\right] \\
\times & h_{3}\left(n_{1} T_{s}, n_{2} T_{s}, n_{3} T_{s}, k_{1}+k_{2}-k_{3}-k\right) \\
\times & h_{3}^{*}\left(n_{1}^{\prime} T_{s}, n_{2}^{\prime} T_{s}, n_{3}^{\prime} T_{s}, k_{1}^{\prime}+k_{2}^{\prime}-k_{3}^{\prime}-k\right) .
\end{aligned}
$$

A lot of indexes can be removed in above equation by remarking that the terms dealing with the symbol expectation are non-null only if both first indexes are equal to each other and if both second indexes are equal to each others. We thus have $k_{1}=k_{1}^{\prime}, k_{2}=k_{3}, k_{2}^{\prime}=k_{3}^{\prime}$ and $n_{1}=n_{1}^{\prime}, n_{2}=n_{3}$, $n_{2}^{\prime}=n_{3}^{\prime}$. Consequently, we get

$$
\begin{aligned}
p_{\mathrm{NL}}^{(1)}(k) & =\gamma_{3}^{2} \sum_{k_{1}, k_{2}, k_{2}^{\prime}=1}^{K} \sum_{n_{1}, n_{2}, n_{2}^{\prime} \in \mathbb{Z}} G_{k_{1}} G_{k_{2}} G_{k_{2}^{\prime}} P_{k_{1}} P_{k_{2}} P_{k_{2}^{\prime}} \\
& \times h_{3}\left(n_{1} T_{s}, n_{2} T_{s}, n_{2} T_{s}, k_{1}-k\right) \\
& \times h_{3}^{*}\left(n_{1} T_{s}, n_{2}^{\prime} T_{s}, n_{2}^{\prime} T_{s}, k_{1}-k\right) .
\end{aligned}
$$

According to [3], the interference coming from the subband is non-negligible only for the current subband and its adjacent subbands. Consequently, only $\left(k_{1}-k\right) \in\{-1,0,1\}$ are considered. Therefore, Eq. (20) can be also split into three terms

$$
p_{\mathrm{NL}}^{(1)}(k)=p_{\mathrm{NL}, 0}^{(1)}(k)+p_{\mathrm{NL}, 1}^{(1)}(k)+p_{\mathrm{NL},-1}^{(1)}(k) .
$$

Each term $p_{\mathrm{NL}, \mathrm{j}}^{(1)}(k)$ is given below:

- Case $k_{1}-k=0$ (intra-band interference). We easily have

$$
p_{\mathrm{NL}, 0}^{(1)}(k)=\gamma_{3}^{2} \alpha^{(1)} G_{k} P_{k} \sum_{k^{\prime}, k^{\prime \prime}=1}^{K} G_{k^{\prime}} G_{k^{\prime \prime}} P_{k^{\prime}} P_{k^{\prime \prime}}
$$

with

$$
\begin{aligned}
\alpha^{(1)} & =\sum_{\substack{n_{1}, n_{2}, n_{3} \in \mathbb{Z} \\
h_{3}^{*}}} h_{3}\left(n_{1} T_{s}, n_{2} T_{s}, n_{2} T_{s}, 0\right) \\
& \left.\times n_{s}, n_{3} T_{s}, n_{3} T_{s}, 0\right) .
\end{aligned}
$$

Actually the term $\alpha^{(1)}$ is positive. Indeed one can prove by swapping adequately indexes in the various sums that

$$
\alpha^{(1)}=\sum_{n^{\prime} \in \mathbb{Z}}\left|\sum_{n^{\prime \prime} \in \mathbb{Z}} h_{3}\left(n^{\prime} T_{s}, n^{\prime \prime} T_{s}, n^{\prime \prime} T_{s}, 0\right)\right|^{2} .
$$

- Case $k_{1}-k=1$ (right adjacent subband interference). We have for $k \neq K$

$$
p_{\mathrm{NL}, 1}^{(1)}(k)=\gamma_{3}^{2} \beta^{(1)} G_{k+1} P_{k+1} \sum_{k^{\prime}, k^{\prime \prime}=1}^{K} G_{k^{\prime}} G_{k^{\prime \prime}} P_{k^{\prime}} P_{k^{\prime \prime}}
$$

with

$$
\begin{aligned}
\beta^{(1)} & =\sum_{\substack{n_{1}, n_{2}, n_{3} \in \mathbb{Z}\\
}} h_{3}\left(n_{1} T_{s}, n_{2} T_{s}, n_{2} T_{s}, 1\right) \\
& \times h_{3}^{*}\left(n_{1} T_{s}, n_{3} T_{s}, n_{3} T_{s}, 1\right) .
\end{aligned}
$$

By using the same argument as for $\alpha^{(1)}$, we see that $\beta^{(1)}$ is positive and equal to

$$
\beta^{(1)}=\sum_{n^{\prime} \in \mathbb{Z}}\left|\sum_{n^{\prime \prime} \in \mathbb{Z}} h_{3}\left(n^{\prime} T_{s}, n^{\prime \prime} T_{s}, n^{\prime \prime} T_{s}, 1\right)\right|^{2} .
$$

The case $k=K$ leads to null term since the last user can not be disturbed by its right as it is empty.

- Case $k_{1}-k=-1$ (left adjacent subband interference). Similarly to the case $k_{1}-k=1$, we have for $k \neq 1$

$$
p_{\mathrm{NL},-1}^{(1)}(k)=\gamma_{3}^{2} \beta^{(1)} G_{k-1} P_{k-1} \sum_{k^{\prime}, k^{\prime \prime}=1}^{K} G_{k^{\prime}} G_{k^{\prime \prime}} P_{k^{\prime}} P_{k^{\prime \prime}}
$$


Notice that $\beta^{(1)}$ is still involved since $h_{3}\left(n_{1} T s, n_{2} T_{s}, n_{3} T_{s},-1\right)=h_{3}^{*}\left(n_{1} T_{s}, n_{2} T_{s}, n_{3} T_{s}, 1\right)$.

The case $k=1$ leads to null term since the first user can not be disturbed by its left as it is empty.

b) Derivations of $p_{\mathrm{NL}}^{(2)}(k)$ : According to Eqs. (17)-(16), we have

$$
\begin{aligned}
p_{\mathrm{NL}}^{(2)}(k)= & \gamma_{3}^{2} \sum_{k_{1}, k_{2}, k_{3}=1}^{K} \sum_{k_{1}^{\prime}, k_{2}^{\prime}, k_{3}^{\prime}=1}^{K} \sum_{n_{1}, n_{2}, n_{3} \in \mathbb{Z}} \sum_{n_{1}^{\prime}, n_{2}^{\prime}, n_{3}^{\prime} \in \mathbb{Z}} \\
& \sqrt{G_{k_{1}} G_{k_{1}^{\prime}} G_{k_{2}} G_{k_{2}^{\prime}} G_{k_{3}} G_{k_{3}^{\prime}}} \\
\times & e^{2 i \pi\left(k_{1}+k_{2}-k_{3}\right) \Delta F n T_{s}} e^{-2 i \pi\left(k_{1}^{\prime}+k_{2}^{\prime}-k_{3}^{\prime}\right) \Delta F n T_{s}} \\
\times & \mathbb{E}\left[a_{k_{1}, n-n_{1}} a_{k_{1}^{\prime}, n-n_{1}^{\prime}}^{*}\right] \mathbb{E}\left[a_{k_{2}, n-n_{2}} a_{k_{2}^{\prime}, n-n_{2}^{\prime}}^{*}\right] \\
\times & \mathbb{E}\left[a_{k_{3}, n-n_{3}}^{*} a_{k_{3}^{\prime}, n-n_{3}^{\prime}}\right] \\
\times & h_{3}\left(n_{1} T_{s}, n_{2} T_{s}, n_{3} T_{s}, k_{1}+k_{2}-k_{3}-k\right) \\
& \times h_{3}^{*}\left(n_{1}^{\prime} T_{s}, n_{2}^{\prime} T_{s}, n_{3}^{\prime} T_{s}, k_{1}^{\prime}+k_{2}^{\prime}-k_{3}^{\prime}-k\right),
\end{aligned}
$$

As previously, only relevant indexes in the symbol expectation are kept. Consequently, $k_{1}=k_{1}^{\prime}, k_{2}=k_{2}^{\prime}, k_{3}=k_{3}^{\prime}$ and $n_{1}=n_{1}^{\prime}, n_{2}=n_{2}^{\prime}, n_{3}=n_{3}^{\prime}$. This implies

$$
\begin{aligned}
p_{\mathrm{NL}}^{(2)}(k) & =\gamma_{3}^{2} \sum_{k_{1}, k_{2}, k_{3}=1}^{K} \sum_{n_{1}, n_{2}, n_{3} \in \mathbb{Z}} G_{k_{1}} G_{k_{2}} G_{k_{3}} P_{k_{1}} P_{k_{2}} P_{k_{3}} \\
& \times\left|h_{3}\left(n_{1} T_{s}, n_{2} T_{s}, n_{3} T_{s}, k_{1}+k_{2}-k_{3}-k\right)\right|^{2} .
\end{aligned}
$$

As previously, by keeping only the terms such that $\left(k_{1}+\right.$ $\left.k_{2}-k_{3}-k\right) \in\{-1,0,1\}$, we have

$$
p_{\mathrm{NL}}^{(2)}(k)=p_{\mathrm{NL}, 0}^{(2)}(k)+p_{\mathrm{NL}, 1}^{(2)}(k)+p_{\mathrm{NL},-1}^{(2)}(k) .
$$

with

- Case $k_{1}+k_{2}-k_{3}-k=0$. We have

$$
p_{\mathrm{NL}, 0}^{(2)}(k)=\gamma_{3}^{2} \alpha^{(2)} \sum_{\substack{k_{1}, k_{2}, k_{3}=1 \\ k=k_{1}+k_{2}-k_{3}}}^{K} G_{k_{1}} G_{k_{2}} G_{k_{3}} P_{k_{1}} P_{k_{2}} P_{k_{3}}
$$

with

$$
\alpha^{(2)}=\sum_{n_{1}, n_{2}, n_{3} \in \mathbb{Z}}\left|h_{3}\left(n_{1} T_{s}, n_{2} T_{s}, n_{3} T_{s}, 0\right)\right|^{2} .
$$

Notice that $\alpha^{(2)}$ is positive and also that the condition on $\left(k_{1}, k_{2}, k_{3}\right)$ in the sum is described in Table 1 of [3].

- Case $k_{1}+k_{2}-k_{3}-k=1$. We have

$$
p_{\mathrm{NL}, 1}^{(2)}(k)=\gamma_{3}^{2} \beta^{(2)} \sum_{\substack{k_{1}, k_{2}, k_{3}=1 \\ k=k_{1}+k_{2}-k_{3}-1}}^{K} G_{k_{1}} G_{k_{2}} G_{k_{3}} P_{k_{1}} P_{k_{2}} P_{k_{3}}
$$

with

$$
\beta^{(2)}=\sum_{n_{1}, n_{2}, n_{3} \in \mathbb{Z}}\left|h_{3}\left(n_{1} T_{s}, n_{2} T_{s}, n_{3} T_{s},-1\right)\right|^{2} .
$$

Notice that $\beta^{(2)}$ is positive. As previously, the condition on $\left(k_{1}, k_{2}, k_{3}\right)$ in the sum is described in Table 2 of [3].

- Case $k_{1}+k_{2}-k_{3}-k=-1$. Similarly, we have

$$
p_{\mathrm{NL},-1}^{(2)}(k)=\gamma_{3}^{2} \beta^{(2)} \sum_{\substack{k_{1}, k_{2}, k_{3}=1 \\ k=k_{1}+k_{2}-k_{3}+1}}^{K} G_{k_{1}} G_{k_{2}} G_{k_{3}} P_{k_{1}} P_{k_{2}} P_{k_{3}} \text {. }
$$

c) Derivations of $p_{\mathrm{NL}}^{(j)}(k)$ for $j=3, \cdots, 6$ : By doing similar derivations, one can easily check that

$$
p_{\mathrm{NL}}^{(1)}(k)=p_{\mathrm{NL}}^{(3)}(k)=p_{\mathrm{NL}}^{(4)}(k)=p_{\mathrm{NL}}^{(5)}(k)
$$

and

$$
p_{\mathrm{NL}}^{(2)}(k)=p_{\mathrm{NL}}^{(6)}(k) .
$$

By encompassing all the previous expressions, we obtain the following final result for $\mathcal{P}_{\mathrm{NL}}(k)$,

$$
\begin{aligned}
\mathcal{P}_{\mathrm{NL}}(k) & =4 \gamma_{3}^{2} \alpha^{(1)} G_{k} P_{k} \sum_{k^{\prime}, k^{\prime \prime}=1}^{K} G_{k^{\prime}} G_{k^{\prime \prime}} P_{k^{\prime}} P_{k^{\prime \prime}} \\
& +2 \gamma_{3}^{2} \alpha^{(2)} \sum_{\substack{k_{1}, k_{2}, k_{3}=1 \\
k=k_{1}+k_{2}-k_{3}}}^{K} G_{k_{1}} G_{k_{2}} G_{k_{3}} P_{k_{1}} P_{k_{2}} P_{k_{3}} \\
& +4 \gamma_{3}^{2} \beta^{(1)}\left(\tilde{\delta}_{k, 1} G_{k-1} P_{k-1}+\tilde{\delta}_{k, K} G_{k+1} P_{k+1}\right) \\
& \times \sum_{k^{\prime}, k^{\prime \prime}=1}^{K} G_{k^{\prime}} G_{k^{\prime \prime}} P_{k^{\prime}} P_{k^{\prime \prime}} \\
& +2 \gamma_{3}^{2} \beta^{(2)} \sum_{\substack{k_{1}, k_{2}, k_{3}=1 \\
k=k_{1}+k_{2}-k_{3} \pm 1}}^{K} G_{k_{1}} G_{k_{2}} G_{k_{3}} P_{k_{1}} P_{k_{2}} P_{k_{3}}
\end{aligned}
$$

where $\tilde{\delta}_{k, k^{\prime}}=1-\delta_{k, k^{\prime}}$ with the Kronecker index $\delta_{k, k^{\prime}}$. Notice that $\mathcal{P}_{\mathrm{NL}}(k)$ is a sum of positive terms.

\section{Derivations of $\mathcal{P}_{\mathrm{LNL}}(k)$}

According ot Eqs. (10) and (11), we have

$$
\begin{aligned}
\mathcal{P}_{\mathrm{LNL}}(k) & =\gamma_{1} \gamma_{3} \sum_{k_{1}, k_{2}, k_{3}=1}^{K} \sum_{n_{1}, n_{2}, n_{3} \in \mathbb{Z}} \\
& \times \sqrt{G_{k} G_{k_{1}} G_{k_{2}} G_{k_{3}}} e^{-2 i \pi\left(k_{1}+k_{2}-k_{3}-k\right) \Delta F n T_{s}} \\
& \times \mathbb{E}\left[a_{k, n} a_{k_{1}, n-n_{1}}^{*} a_{k_{2}, n-n_{2}}^{*} a_{k_{3}, n-n_{3}}\right] \\
& \times h_{3}^{*}\left(n_{1} T_{s}, n_{2} T_{s}, n_{3} T_{s}, k_{1}+k_{2}-k_{3}-k\right) .
\end{aligned}
$$

Following the same derivation steps than for $\mathcal{P}_{\mathrm{NL}}(k)$ (i.e. first decompose the fourth-order term in $\left\{a_{k, n}\right\}_{k, n}$, then looking at the simplification on $h_{3}$ ), we obtain

$$
\mathcal{P}_{\mathrm{LNL}}(k)=2 \gamma_{1} \gamma_{3} \lambda G_{k} P_{k} \sum_{k^{\prime}=1}^{K} G_{k^{\prime}} P_{k^{\prime}}
$$

with $\lambda=\sum_{n_{1} \in \mathbb{Z}} h_{3}^{*}\left(0, n_{1} T_{s}, n_{1} T_{s}, 0\right)$. Using the symmetric of the RRC filter, one can prove that $\lambda$ is positive.

Thanks to these closed-form expressions, two remarks can be done: $\mathcal{P}_{\mathrm{LNL}}(k)$ is a positive term which implies that it enables us to increase the capacity given by Eq. (13). In addition, each subband has significant impact on $\mathcal{P}_{\mathrm{NL}}(k)$ and $\mathcal{P}_{\mathrm{LNL}}(k)$ since the terms related to subband $k^{\prime}$ are not multiplied by negligible weights. 


\section{NUMERICAL RESULTS}

A multiband single-beam satellite is considered. The system operates in the Ka-band for the uplink $(27.5-29.5 \mathrm{GHz})$ and $K=6$ terrestrial users are in the beam coverage. The number of subbands is equal to $K$, and the subband assignment has been already performed [7]. The channel gains $\{G(k)\}_{k}$ are computed according to [8]. The roll-off of RRC filters is 0.25 . The values $\gamma_{1}$ and $\gamma_{3}$ are 1 and 0.05 respectively.

In Fig. 1, we plot $C, \underline{C}$ and the capacity for Gaussian channel (expressed by $\left.C_{\mathrm{AWGN}}(k)=\log _{2}\left(1+\mathcal{P}_{\mathrm{L}}(k) / \mathcal{P}_{\mathrm{W}}\right)\right)$ versus the transmit power of the terrestrial users (we assume $P_{k}=P_{k^{\prime}}=P$ for any $\left.k, k^{\prime}\right)$. We have consider three

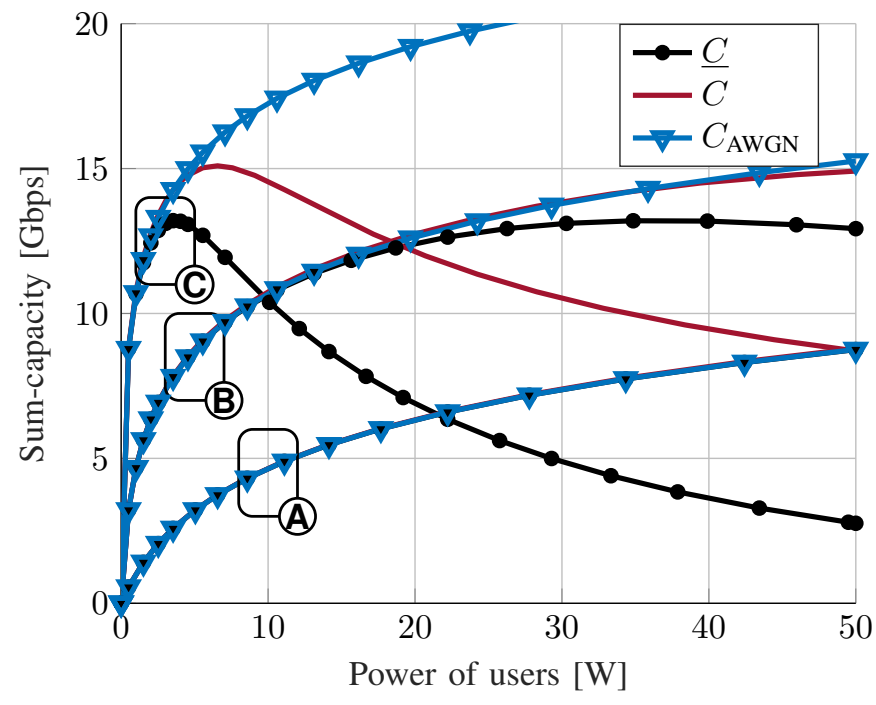

Fig. 1. Sum-capacity vs. user's power $P$ for $K=6$ and 3 different values of pre-amplifier gain $G_{\mathrm{amp}} \in\{-10,0,+10\} \mathrm{dB}$ (A, B and C respectively).

configurations: each of them corresponds to a satellite preamplifier gain value. Actually, this device is located before HPA which operates into linear or nonlinear mode depending on the tuning of the pre-amplifier. Here A, B, and C deal with linear, transient and nonlinear modes respectively. In nonlinear mode, we show that i) the decoder related to $C$ offers a gain of $5 \%$ if the power is chosen to maximize $\underline{C}$ and ii) the power to operate is different according to the selected decoder and the gain with $C$ is then around $12 \%$ if the power is well tuned.

In Fig. 2, we plot $C, \underline{C}$, and $C_{\mathrm{AWGN}}$ versus the pre-amplifier gain for two weather conditions (D: clear sky; E: rain for a third of users) and two power allocations. In the first one (called "naive"), we force all the users to have the same power (ie. $P_{k}=P_{k^{\prime}}$ ) and we select the power within the interval $\left[0, P_{\max }\right]$ maximizing the sum-capacity. The algorithm relies on a 1-D search. In the second one (called "exhaustive"), we allow users to have their own power within $\left[0, P_{\max }\right]$. So we may have $P_{k} \neq P_{k^{\prime}}$ and a $K$-D search is applied.

The difference between power allocations occurs on the nonlinear regime. Indeed in linear regime, the users use their maximum power since they do not interact to each other. Under (D), the gain of the exhaustive allocation is marginal

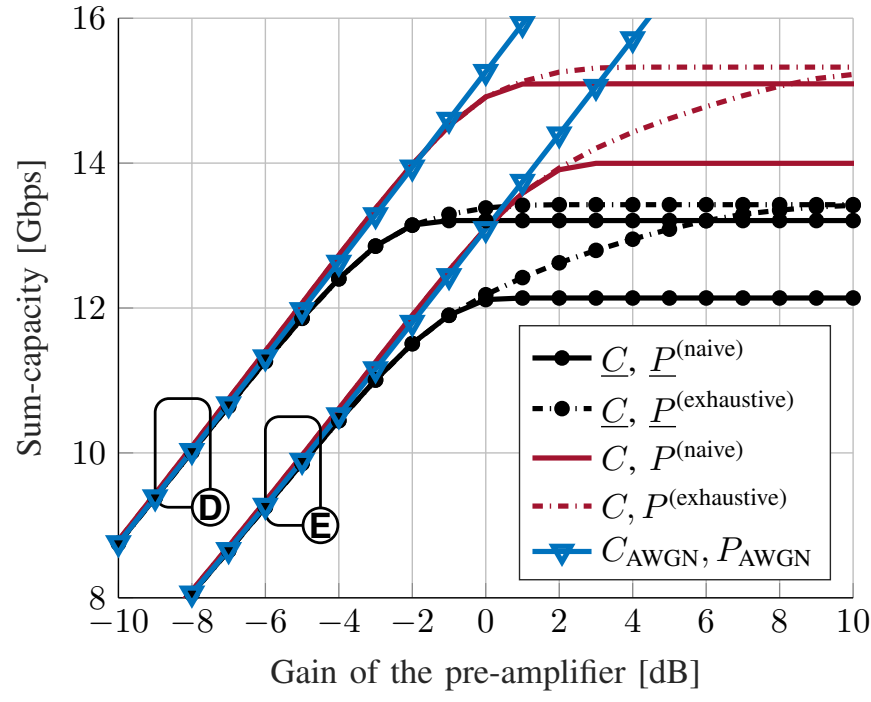

Fig. 2. Sum-capacity vs. pre-amplifier gain $G_{\mathrm{amp}}$ for $K=6$ and $P_{\max }=$ $50 \mathrm{~W}$. (D) corresponds to clear sky while (E) corresponds to rain for two users.

(around 2\%). Under (E), the gain is more significant (around $8 \%$ ) since $G_{k}$ are quite different amongst users. Notice that this configuration is realistic as the beam covers a large area on Earth which may lead to different weather conditions.

\section{CONCLUSION}

In the context of uplink multiband satellite communications, we have provided closed-form expression for the capacity in nonlinear regime generated by the satellite amplifier. In future works, we plan to take into account the time and frequency correlations for the capacity derivations and to design practical algorithms for the power allocations instead of just a prohibitive exhaustive search.

\section{REFERENCES}

[1] S. Maleki, S. Chatzinotas, B. Evans, K. Liolis, J. Grotz, A. VanelliCoralli, and N. Chuberre, "Cognitive spectrum utilization in ka band multibeam satellite communications," IEEE Communications Magazine, vol. 53, no. 3, pp. 24-29, 2015

[2] T. Deleu, M. Dervin, and F. Horlin, "Low complexity block pre-distortion of a multi-carrier non-linear satellite channel," in IEEE International Conference on Communications (ICC), 2014, pp. 4325-4330.

[3] B. F. Beidas, "Intermodulation distortion in multicarrier satellite systems: Analysis and turbo volterra equalization," IEEE Transactions on Communications, vol. 59, no. 6, pp. 1580-1590, 2011.

[4] B. Stephen, K. Seung-Jean, V. Lieven, and H. Arash, "A tutorial on geometric programming," Optimization and Engineering, vol. 8, no. 1, p. 67, 2007.

[5] R. A. Ince, B. L. Giordano, C. Kayser, G. A. Rousselet, J. Gross, and P. G. Schyns, "A statistical framework for neuroimaging data analysis based on mutual information estimated via a gaussian copula," Human Brain Mapping, vol. 38, no. 3, pp. 1541-1573, 2017.

[6] P. Delesques, "Analyses of transmission and switching capacities in optical networks," Ph.D. dissertation, Telecom Paris, December 2012

[7] A. Louchart, P. Ciblat, and P. d. Kerret, "Resource optimization for $\operatorname{cog}$ nitive satellite systems with incumbent terrestrial receivers," in European Signal Processing Conference (EUSIPCO), 2019, pp. 1-5.

[8] E. Lagunas, S. K. Sharma, S. Maleki, S. Chatzinotas, and B. Ottersten, "Resource allocation for cognitive satellite communications with incumbent terrestrial networks," IEEE Transactions on Cognitive Communications and Networking, vol. 1, no. 3, pp. 305-317, 2015. 